\title{
Geometric characterization for homeomorphisms between disks
}

by

\author{
SHUlOng Li and LiXIN LIU (Guangzhou)
}

\begin{abstract}
We give some characterizations for certain homeomorphisms between disks in the complex plane, and we prove some Schwarz type theorems for such homeomorphisms. Our results replace the main result of Chen [Studia Math. 157 (2003)] which we show to be false.
\end{abstract}

1. Preliminaries. Let $D$ be a domain in the complex plane and $\Gamma$ be a family of rectifiable arcs in $D$. A function $\varrho$ defined on $D$ is called admissible for $\Gamma$ if it satisfies the following conditions:

(1) $\varrho$ is a non-negative Borel measurable function,

(2) $A(\varrho)=\iint_{D} \varrho^{2} d x d y \neq 0, \infty$.

Any $\gamma \in \Gamma$ has a well-defined $\varrho$ length

$$
L_{\gamma}(\varrho)=\int_{\gamma} \varrho|d z|
$$

which may be infinite. Let

$$
L(\varrho)=\inf _{\gamma \in \Gamma} L_{\gamma}(\varrho) .
$$

The extremal length of $\Gamma$ is defined to be

$$
\lambda(\Gamma)=\sup _{\varrho} \frac{L(\varrho)^{2}}{A(\varrho)},
$$

where the supremum is taken over all admissible $\varrho$.

Let $Q$ denote a (topological) quadrilateral, that is, a Jordan domain in the complex plane with four distinguished boundary points which divide the boundary curve into four arcs, the sides of $Q$. Then $Q$ can be mapped conformally onto a rectangle $Q^{\prime}$ with sides of lengths $a$ and $b$ so that vertices

2000 Mathematics Subject Classification: 30C62, 30C55.

Key words and phrases: quasiconformal mapping, extremal length, affine mapping.

The research was supported by the NNSF Grant of China and the Foundation of Natural Science of Guangdong Province. 
map to vertices. Consider the pair of opposite sides of $Q$ which correspond to the sides of $Q^{\prime}$ of length $a$. The modulus of $Q$ with respect to this pair of sides is defined as $a / b$, which is the extremal length of the curve family in this rectangle connecting the other two sides. The modulus of $Q$ with respect to the other pair is then $b / a$. We denote either of these moduli by $\bmod Q$.

Define the modulus mod $A\left(r_{1}, r_{2}\right)$ of an annulus

$$
A\left(r_{1}, r_{2}\right)=\left\{z\left|r_{1}<\right| z \mid<r_{2}\right\}
$$

as the extremal length of the family $\Gamma$ of arcs that connect $\left\{z|| z \mid=r_{1}\right\}$ and $\left\{z|| z \mid=r_{2}\right\}$ in $A\left(r_{1}, r_{2}\right)$. Then

$$
\bmod A\left(r_{1}, r_{2}\right)=\frac{1}{2 \pi} \log \frac{r_{2}}{r_{1}} .
$$

By the conformal invariance of extremal lengths, we can define the modulus of any ring domain.

Define the modulus $\bmod A\left(r_{1}, r_{2} ; \theta_{1}, \theta_{2}\right)$ of a sector

$$
A\left(r_{1}, r_{2} ; \theta_{1}, \theta_{2}\right)=\left\{z\left|r_{1}<\right| z \mid<r_{2} ; \theta_{1}<\arg z<\theta_{2}\right\}
$$

as the extremal length of the family $\Gamma$ of $\operatorname{arcs}$ that connect $\left\{z|| z \mid=r_{1}\right\}$ and $\left\{z|| z \mid=r_{2}\right\}$ in $A\left(r_{1}, r_{2} ; \theta_{1}, \theta_{2}\right)$. Then

$$
\bmod A\left(r_{1}, r_{2} ; \theta_{1}, \theta_{2}\right)=\frac{1}{\theta_{2}-\theta_{1}} \log \frac{r_{2}}{r_{1}} .
$$

By the continuity of modulus, we can view an annulus as a sector with $\theta_{1}=0$ and $\theta_{2}=2 \pi$.

A sense-preserving homeomorphism $\omega$ of a domain $G$ in the complex plane onto a domain $G^{\prime}$ in the complex plane is said to be $K$-quasiconformal if

$$
\bmod \omega(Q) \leq K \bmod Q
$$

for each quadrilateral $Q$ whose closure $\bar{Q}$ lies in $G$, where the moduli are taken with respect to the corresponding pair of sides. This is called the geometric definition of $K$-quasiconformal mappings.

It is natural to look for other geometric definitions of quasiconformal mappings. In [4], Gehring and Väisälä showed that the quadrilaterals in the above definition can be replaced by rectangles. If the quadrilaterals are replaced by annuli or oriented rectangles, we obtain a $\left(K+\sqrt{K^{2}-1}\right)$ quasiconformal mapping. Here an oriented rectangle is a rectangle with a pair of its sides parallel to some fixed line. In this paper we consider a similar question and we show that for homeomorphisms between unit disks, the quadrilaterals in the above cannot be replaced by concentric sectors.

It is interesting to generalize the Schwarz lemma to quasiconformal mappings or other homeomorphisms between disks in the complex plane. Let $\Delta$ be the unit disk in the complex plane. In [7], Zhu, Zhou and He obtained the following Schwarz type theorem. 
THEOREM A. If $K \geq 1$ and $f(z)$ is a K-quasiconformal mapping of $\Delta$ onto itself which satisfies $f(0)=0$ and

$$
\lim _{z \rightarrow 0} \frac{|f(z)|}{|z|^{1 / K}}=1
$$

then $f(z)=\lambda z|z|^{1 / K-1}$ for any $z \in \Delta$, where $\lambda$ is a constant with $|\lambda|=1$.

One can ask if it is possible to obtain the same conclusion under weaker conditions. Chen stated in [3] some results of this type. His main statement (Theorem 2.1 in [3]) is:

THEOREM B. Let $f(z)$ be a sense-preserving homeomorphism of the unit disk $\Delta$ onto itself with normalization $f(0)=0$. If

$$
\frac{1}{K} \bmod A \leq \bmod f(A)
$$

for any $A=A\left(r_{1}, r_{2}\right) \subset \Delta$ and $A=A\left(r_{1}, r_{2} ; \theta_{1}, \theta_{2}\right) \subset \Delta$, and

$$
\lim _{z \rightarrow 0} \frac{|f(z)|}{|z|^{1 / K}}=1
$$

then $f(z)=\lambda z|z|^{1 / K-1}$ for any $z \in \Delta$, where $\lambda$ is a constant with $|\lambda|=1$.

Unfortunately, our Examples 1 and 2 show that this statement is false. We prove the following related result:

THEOREM 1. Let $K \geq 1$ and $f(z)$ be a sense-preserving homeomorphism of the unit disk $\Delta$ onto itself with $f(0)=0$. If

$$
\bmod A \leq K \bmod f(A) \quad \text { for any annulus } A=A\left(r_{1}, r_{2}\right) \subset \Delta \text {, }
$$

and

$$
\lim _{z \rightarrow 0} \frac{|f(z)|}{|z|^{1 / K}}=1,
$$

then $|f(z)|=|z|^{1 / K}$ for any $z \in \Delta$.

We also give a sufficient condition for the conclusion of Theorem B to be valid.

TheOREM 2. Let $K \geq 1$ and $f(z)$ be a sense-preserving homeomorphism of the unit disk $\Delta$ onto itself with $f(0)=0$. If

$\bmod A \leq K \bmod f(A) \quad$ for any annulus $A=A\left(r_{1}, r_{2}\right) \subset \Delta$,

$$
\lim _{z \rightarrow 0} \frac{|f(z)|}{|z|^{1 / K}}=1
$$

and

(4) $\bmod f(A) \leq \frac{1}{K} \bmod A \quad$ for any $A=A\left(r_{1}, r_{2} ; \theta_{1}, \theta_{2}\right) \subset \Delta$, then $f(z)=\lambda z|z|^{1 / K-1}$ for any $z \in \Delta$, where $\lambda$ is a constant and $|\lambda|=1$. 
We answer a weak form of a problem suggested by Chen in [3]:

TheOREM 3. Let $K \geq 1$ and $f(z)$ be a sense-preserving homeomorphism from $D_{1}=\{z|r \leq| z \mid \leq 1\}$ onto $D_{2}=\left\{z\left|r^{K} \leq\right| z \mid \leq 1\right\}$. If $\bmod f(A) \leq K \bmod A \quad$ for any $A=A\left(r_{1}, r_{2} ; \theta_{1}, \theta_{2}\right) \subset D_{1}$, then $f(z)=\lambda z|z|^{K-1}$ for any $z \in D_{1}$, where $\lambda$ is a constant with $|\lambda|=1$.

In the last part of this paper we discuss some related problems.

2. Auxiliary lemmas. We give some well-known lemmas first.

Lemma 1 ([1], [2], [6]). If every $\gamma \in \Gamma$ contains some $\gamma^{\prime} \in \Gamma^{\prime}$, then $\lambda(\Gamma)$ $\geq \lambda\left(\Gamma^{\prime}\right)$.

Lemma 2 ([1], [2], [6]). Let $D_{1}$ and $D_{2}$ be two disjoint domains in the complex plane, let $\Gamma_{1}$ and $\Gamma_{2}$ consist of rectifiable arcs in $D_{1}, D_{2}$, respectively, and let $\Gamma$ be a third family of rectifiable arcs.

(1) If every $\gamma \in \Gamma$ contains some $\gamma_{1} \in \Gamma_{1}$ and $\gamma_{2} \in \Gamma_{2}$, then

$$
\lambda(\Gamma) \geq \lambda\left(\Gamma_{1}\right)+\lambda\left(\Gamma_{2}\right) .
$$

(2) If every $\gamma_{1} \in \Gamma_{1}$ and every $\gamma_{2} \in \Gamma_{2}$ contains some $\gamma \in \Gamma$, then

$$
\frac{1}{\lambda(\Gamma)} \geq \frac{1}{\lambda\left(\Gamma_{1}\right)}+\frac{1}{\lambda\left(\Gamma_{2}\right)}
$$

Lemma 3 ([1], [2], [6]). Let $D_{1}$ and $D_{2}$ be two disjoint domains in $A\left(r_{1}, r_{2} ; \theta_{1}, \theta_{2}\right)$. Let $\Gamma_{1}$ and $\Gamma_{2}$ consist of rectifiable arcs in $D_{1}, D_{2}$, respectively, and let $\Gamma$ be the family of rectifiable arcs that connect $\left\{z|| z \mid=r_{1}\right\}$ and $\left\{z|| z \mid=r_{2}\right\}$ in $A\left(r_{1}, r_{2} ; \theta_{1}, \theta_{2}\right)$.

(1) If every $\gamma \in \Gamma$ contains some $\gamma_{1} \in \Gamma_{1}$ and $\gamma_{2} \in \Gamma_{2}$, then

$$
\lambda(\Gamma)=\lambda\left(\Gamma_{1}\right)+\lambda\left(\Gamma_{2}\right) .
$$

if and only if one of $D_{k}, k=1,2$, is $A\left(r_{1}, r ; \theta_{1}, \theta_{2}\right)$ and the other is $A\left(r, r_{2} ; \theta_{1}, \theta_{2}\right)$, for some $r_{1} \leq r \leq r_{2}$.

(2) If every $\gamma_{1} \in \Gamma_{1}$ and every $\gamma_{2} \in \Gamma_{2}$ contains some $\gamma \in \Gamma$, then

$$
\frac{1}{\lambda(\Gamma)}=\frac{1}{\lambda\left(\Gamma_{1}\right)}+\frac{1}{\lambda\left(\Gamma_{2}\right)}
$$

if and only if one of $D_{k}, k=1,2$, is $A\left(r_{1}, r_{2} ; \theta_{1}, \theta\right)$ and the other is $A\left(r_{1}, r_{2} ; \theta, \theta_{2}\right)$, for some $\theta_{1} \leq \theta \leq \theta_{2}$.

3. Proofs of the main results. Theorem 1 will follow from Lemmas 4 and 5 below, which were proved in [3]. For the sake of completeness, we repeat their proofs here.

Define

$$
R(r)=\{z|r<| z \mid<1\}
$$


Then there exists a conformal mapping $\Phi_{r}$ such that

$$
\Phi_{r} \circ f(R(r))=\{\zeta|\phi(r)<| \zeta \mid<1\} .
$$

Set $g_{r}=\Phi_{r} \circ f$.

For any set $V$ in the complex plane, let int $V$ and $\bar{V}$ stand for the interior and the closure of $V$.

LEMMA 4. If $f(z)$ satisfies condition (3), then $\phi(r) / r^{1 / K}$ is an increasing function on $(0,1]$ and $\phi(r) / r^{1 / K} \leq 1$.

Proof. For $0<r_{1}<r_{2} \leq 1$, set $A=\left\{z\left|r_{1}<\right| z \mid<r_{2}\right\}, B=\left\{z \mid r_{2}<\right.$ $|z|<1\}, A^{\prime}=f(A), B^{\prime}=f(B)$. By Lemma 2,

$$
\bmod A^{\prime}+\bmod B^{\prime} \leq \bmod \left(\operatorname{int}\left(\overline{A^{\prime}} \cup \overline{B^{\prime}}\right)\right) .
$$

By conformal invariance of modulus,

$$
\begin{gathered}
\bmod B^{\prime}=\bmod f(B)=\bmod g_{r_{2}}(B)=\frac{1}{2 \pi} \log \frac{1}{\phi\left(r_{2}\right)}, \\
\bmod \left(\operatorname{int}\left(\overline{A^{\prime}} \cup \overline{B^{\prime}}\right)\right)=\bmod f(\operatorname{int}(\bar{A} \cup \bar{B}))=\bmod g_{r_{1}}(\operatorname{int}(\bar{A} \cup \bar{B})) \\
=\frac{1}{2 \pi} \log \frac{1}{\phi\left(r_{1}\right)} .
\end{gathered}
$$

By (3),

$$
\bmod A \leq K \bmod A^{\prime}
$$

Thus

$$
\frac{1}{2 \pi} \log \frac{r_{2}}{r_{1}} \leq K \bmod A^{\prime} \leq K\left(\bmod \left(\operatorname{int}\left(\overline{A^{\prime}} \cup \overline{B^{\prime}}\right)\right)-\bmod B^{\prime}\right)=\frac{K}{2 \pi} \log \frac{\phi\left(r_{2}\right)}{\phi\left(r_{1}\right)} .
$$

Hence

$$
\log \frac{r_{2}}{r_{1}} \leq K \log \frac{\phi\left(r_{2}\right)}{\phi\left(r_{1}\right)}
$$

and so

$$
\frac{\phi\left(r_{1}\right)}{r_{1}^{1 / K}} \leq \frac{\phi\left(r_{2}\right)}{r_{2}^{1 / K}}
$$

This shows that $\phi(r) / r^{1 / K}$ is increasing on $(0,1]$. Letting $r_{2}=1$ yields $\phi(r) / r^{1 / K} \leq 1$.

LEMMA 5. If $f(z)$ satisfies condition (2), then

$$
\lim _{r \rightarrow 0} \frac{\phi(r)}{r^{1 / K}}=1 \text {. }
$$

Proof. By (2), for any $\varepsilon>0$ there exists $\delta>0$ such that for any $r<\delta$ and $\theta \in[0,2 \pi)$,

$$
1-\varepsilon \leq \frac{\left|f\left(r e^{i \theta}\right)\right|}{r^{1 / K}} \leq 1+\varepsilon .
$$


Hence

$$
(1-\varepsilon) r^{1 / K} \leq\left|f\left(r e^{i \theta}\right)\right| \leq(1+\varepsilon) r^{1 / K},
$$

and so

$$
\left\{w\left|(1+\varepsilon) r^{1 / K}<\right| w \mid<1\right\} \subset f(R(r)) \subset\left\{w\left|(1-\varepsilon) r^{1 / K}<\right| w \mid<1\right\} .
$$

By Lemma 1,

$$
\frac{1}{2 \pi} \log \frac{1}{(1+\varepsilon) r^{1 / K}} \leq \bmod f(R(r)) \leq \frac{1}{2 \pi} \log \frac{1}{(1-\varepsilon) r^{1 / K}} .
$$

By conformal invariance of modulus,

$$
\bmod f(R(r))=\bmod g_{r}(R(r))=\frac{1}{2 \pi} \log \frac{1}{\phi(r)} .
$$

Thus we get the following inequalities:

$$
\frac{1}{2 \pi} \log \frac{1}{(1+\varepsilon) r^{1 / K}} \leq \frac{1}{2 \pi} \log \frac{1}{\phi(r)} \leq \frac{1}{2 \pi} \log \frac{1}{(1-\varepsilon) r^{1 / K}} .
$$

Hence

$$
1-\varepsilon \leq \frac{\phi(r)}{r^{1 / K}} \leq 1+\varepsilon,
$$

completing the proof.

From Lemmas 4 and 5 we know that

$$
\phi(r)=r^{1 / K} \quad \text { for } 0 \leq r \leq 1 .
$$

Proof of Theorem 1. For $0<r_{1}<r_{2} \leq 1$, set $A=\left\{z\left|r_{1}<\right| z \mid<r_{2}\right\}$, $B=\left\{z\left|r_{2}<\right| z \mid<1\right\}, A^{\prime}=f(A), B^{\prime}=f(B), A^{\prime \prime}=\Phi_{r_{1}} \circ f(A), B^{\prime \prime}=$ $\Phi_{r_{1}} \circ f(B)$. Moreover, set $g_{r}=\Phi_{r} \circ f$ for $r \in(0,1)$. Then

$$
\bmod \left(\operatorname{int}\left(\overline{A^{\prime \prime}} \cup \overline{B^{\prime \prime}}\right)\right)=\bmod g_{r_{1}}(\operatorname{int}(\bar{A} \cup \bar{B}))=\frac{1}{2 \pi} \log \frac{1}{\phi\left(r_{1}\right)}=\frac{1}{2 \pi K} \log \frac{1}{r_{1}}
$$

and

$$
\begin{aligned}
\bmod B^{\prime \prime} & =\bmod g_{r_{1}}(B)=\bmod f(B) \\
& =\bmod g_{r_{2}}(B)=\frac{1}{2 \pi} \log \frac{1}{\phi\left(r_{2}\right)}=\frac{1}{2 \pi K} \log \frac{1}{r_{2}} .
\end{aligned}
$$

By conformal invariance of modulus,

$$
\bmod A^{\prime}=\bmod A^{\prime \prime} \text {. }
$$

By (3),

$$
\frac{1}{K} \bmod A \leq \bmod A^{\prime}
$$

and by Lemma 2 ,

$$
\bmod A^{\prime}+\bmod B^{\prime} \leq \bmod \left(\operatorname{int}\left(\overline{A^{\prime}} \cup \overline{B^{\prime}}\right)\right) .
$$


Combining the above facts, we have

$$
\frac{1}{2 \pi K} \log \frac{r_{2}}{r_{1}} \leq \bmod A^{\prime} \leq \frac{1}{2 \pi} \log \frac{\phi\left(r_{2}\right)}{\phi\left(r_{1}\right)}=\frac{1}{2 \pi} \log \left(\frac{r_{2}}{r_{1}}\right)^{1 / K} .
$$

So

$$
\bmod A^{\prime \prime}=\bmod A^{\prime}=\frac{1}{2 \pi K} \log \frac{r_{2}}{r_{1}} .
$$

Thus we know that

$$
\bmod A^{\prime \prime}+\bmod B^{\prime \prime}=\bmod \left(\operatorname{int}\left(\overline{A^{\prime \prime}} \cup \overline{B^{\prime \prime}}\right)\right) .
$$

By Lemma $3, A^{\prime \prime}, B^{\prime \prime}$ are annuli with center at $z=0$. Then

$$
\bmod B^{\prime \prime}=\frac{1}{2 \pi K} \log \frac{1}{r_{2}} \quad \text { implies } \quad B^{\prime \prime}=\left\{z\left|r_{2}^{1 / K}<\right| z \mid<1\right\},
$$

and

$$
\bmod A^{\prime}=\frac{1}{2 \pi K} \log \frac{r_{2}}{r_{1}} \quad \text { implies } \quad A^{\prime \prime}=\left\{z\left|r_{1}^{1 / K}<\right| z \mid<r_{2}^{1 / K}\right\} .
$$

We normalize each $\Phi_{r}$ by $\lim _{z \rightarrow 1} \Phi_{r}(z)=1$ for any $r \in(0,1)$. By definition, we see that $\Phi_{r_{2}}\left(B^{\prime}\right)=B^{\prime \prime}$. Then

$$
\Psi:=\Phi_{r_{2}} \circ \Phi_{r_{1}}^{-1}: B^{\prime \prime} \rightarrow B^{\prime \prime}
$$

is a conformal mapping. We may extend it conformally to a mapping $\Psi_{*}$ of the unit disk $\Delta$ onto itself. Thus $\Psi_{*}$ is a Möbius transformation of $\Delta$. Since $\Psi_{*}(0)=0$ and

$$
\lim _{z \rightarrow 1} \Psi_{*}(z)=\lim _{z \rightarrow 1} \Psi_{*}\left(\Phi_{r_{1}}(z)\right)=\lim _{z \rightarrow 1} \Phi_{r_{2}}(z)=1,
$$

we have $\Psi_{*}(z)=z$ for $z \in \Delta$, and so

$$
\Phi_{r_{2}}(z)=\Phi_{r_{1}}(z), \quad z \in B^{\prime} .
$$

Moreover, $\Phi_{r_{2}} \circ f\left(R\left(r_{2}\right)\right)=R\left(r_{2}^{1 / K}\right)$.

If $r$ tends to 0 , we get a conformal mapping

$$
\Phi_{0}:\{z|0<| z \mid<1\} \rightarrow\{w|0<| w \mid<1\}
$$

with a removable singularity at $z=0$. If we define $\Phi_{0}(0)=0$, then

$$
\Phi_{0}:\{z|| z \mid<1\} \rightarrow\{w|| w \mid<1\}
$$

is conformal and onto with $\Phi_{0}(0)=0$ and $\lim _{z \rightarrow 1} \Phi_{0}(z)=1$, and so

$$
\Phi_{0}(z)=z, \quad z \in \Delta .
$$

Because $\Phi_{0}$ is a conformal extension of $\Phi_{r}$ for any $0 \leq r<1$, it follows that

$$
\Phi_{r}(z)=z, \quad z \in f(R(r)), 0 \leq r<1 .
$$

Thus $f(R(r))=\left\{w\left|r^{1 / K}<\right| w \mid<1\right\}$. So $f$ maps $\{z|| z \mid=r\}$ onto $\left\{w|| w \mid=r^{1 / K}\right\}$, and $\{z|| z \mid \leq r\}$ onto $\left\{w|| w \mid \leq r^{1 / K}\right\}$. This finishes the proof of Theorem 1. 
Now we consider the following problem:

Problem 1. Let $K \geq 1$ and $f(z)$ be a sense-preserving homeomorphism of the unit disk $\Delta$ onto itself, with $f(0)=0$. If

$$
\bmod A \leq K \bmod f(A) \quad \text { for any } A=A\left(r_{1}, r_{2} ; \theta_{1}, \theta_{2}\right) \subset \Delta \text {, }
$$

and

$$
\lim _{z \rightarrow 0} \frac{|f(z)|}{|z|^{1 / K}}=1,
$$

is it true that $f(z)=\lambda z|z|^{1 / K-1}$ for any $z \in \Delta$ ? Here $\lambda$ is a constant with $|\lambda|=1$.

We will give two examples to show that the answer to the above problem is negative.

REMARK. The main result of [3] claims to give a positive answer to the above problem. However, the argument in [3], from page 77, line 8 to page 78 , line -9 , is erroneous because a function of two variables is treated as a function of only one variable.

EXAmple 1. Let $f_{0}: \Delta \rightarrow \Delta$ be given by, for $z=r e^{i \theta} \in \Delta$,

$$
f_{0}\left(r e^{i \theta}\right)=r^{1 / K} e^{i(\theta+t r)}
$$

where $t$ is a constant. This map is obtained as follows: first we take the affine map $f(z)=z|z|^{1 / K-1}$, and then we perform a twist. Because a twist on a sector $A\left(r_{1}, r_{2} ; \theta_{1}, \theta_{2}\right)$ increases its modulus, we see that $f_{0}(z)$ satisfies the conditions of Problem 1. But $f_{0}(z)$ is not affine, so the answer to Problem 1 is negative. By direct computations, we find that the Beltrami coefficient of $f_{0}(z)$ is

$$
\mu_{0}\left(r e^{i \theta}\right)=\frac{(1-K)+K t r i}{(1+K)+K t r i} e^{2 \theta i}
$$

and

$$
0<\frac{K-1}{K+1} \leq\left|\mu_{0}\left(r e^{i \theta}\right)\right| \leq\left(\frac{(1-K)^{2}+K^{2} t^{2}}{(1+K)^{2}+K^{2} t^{2}}\right)^{1 / 2}<1 .
$$

So $f_{0}(z)$ is a quasiconformal mapping of $\Delta$ to itself.

Actually, we can also give examples of mappings which satisfy all the conditions in Problem 1 but they are not even quasiconformal.

If we can find an arc $L$ connecting $(0,0)$ to a point on the unit circle which is not a quasiarc, and the set $\{z|| z \mid=r\} \cap L$ consists of only one point for any $0 \leq r \leq 1$, then we can define $f_{1}: \Delta \rightarrow \Delta$ as follows: first let $R(\theta)$ be the radius connecting $z=0$ to $z=e^{i \theta}, f_{1}(0)=0$. For $z=r, r>0$, let $\left\{f_{1}(r)\right\}$ be equal to $\{z|| z \mid=r\} \cap L$. Thus $f_{1}(R(0))=L$. The image of $R(\theta)$ under $f_{1}$ is obtained by rotating $L=f_{1}(R(0))$ through an angle $\theta$ about $(0,0)$. Thus we obtain a homeomorphism $f_{1}(z)$ of the unit disk. It 
is obvious that $f_{1}(z)$ satisfies all the conditions in Problem 1 for $K=1$. Because $f_{1}(R(0))=L$ and $L$ is not a quasiarc, we find that $f_{1}(z)$ is not quasiconformal.

Next we give an example of an $\operatorname{arc} L$ satisfying the above conditions.

EXAMPLE 2. Let $L$ be a curve in the complex plane which consists of two subcurves $L_{1}$ and $L_{2}$, defined as follows (we sometimes denote the point corresponding to $z=x+i y$ by $(x, y))$ :

$$
\begin{array}{ll}
L_{1}=\left(x_{1}(y), y\right), \quad x_{1}(y)=\left(\frac{1}{16}-\left(y-\frac{1}{4}\right)^{2}\right)^{1 / 2}, \quad 0 \leq y \leq 1 / 2, \\
L_{2}=\left(x_{2}(y), y\right), \quad x_{2}(y)=\left(\frac{1}{16}-\left(y-\frac{3}{4}\right)^{2}\right)^{1 / 2}, \quad 1 / 2 \leq y \leq 1 .
\end{array}
$$

Direct computations show that the point $(0,1 / 2)$ on $L$ is a cusp point. Thus $L$ is not a quasiarc. Moreover, $L$ satisfies all the desired assumptions, so using it we can construct a map $f_{1}: \Delta \rightarrow \Delta$ that satisfies the conditions of Problem 1 and is not even quasiconformal.

Proof of Theorem 2. Without loss of generality, we assume that $f(1)=1$. By Theorem 1, we know that for any $z \in \Delta,|f(z)|=|z|^{1 / K}$. Fix $r \in(0,1)$ and set $A=A(r, 1 ; 0, \theta), B=A(r, 1 ; \theta, 2 \pi), A_{1}=f(A), B_{1}=f(B)$. Then by Lemma 2 ,

$$
\begin{aligned}
\frac{2 K \pi}{\log \frac{1}{r}} & =\frac{K}{\bmod A}+\frac{K}{\bmod B} \leq \frac{1}{\bmod A_{1}}+\frac{1}{\bmod B_{1}} \\
& \leq \frac{1}{\bmod \left(\operatorname{int}\left(\bar{A}_{1} \cup \bar{B}_{1}\right)\right)}=\frac{2 K \pi}{\log \frac{1}{r}} .
\end{aligned}
$$

Hence

$$
\frac{1}{\bmod A_{1}}+\frac{1}{\bmod B_{1}}=\frac{1}{\bmod \left(\operatorname{int}\left(\bar{A}_{1} \cup \bar{B}_{1}\right)\right)},
$$

as well as

$$
\bmod A_{1}=\frac{\bmod A}{K} \text { and } \bmod B_{1}=\frac{\bmod B}{K} .
$$

Thus by Lemma $3, A_{1}=A\left(r^{1 / K}, 1 ; 0, \theta\right)$ and $B_{1}=A\left(r^{1 / K}, 1 ; \theta, 2 \pi\right)$. Hence $f(z)=\lambda z|z|^{1 / K-1}$, where $\lambda$ is a constant and $|\lambda|=1$.

From Example 1, we know that condition (4) is necessary for the conclusion to hold.

COROLlary 1. Let $K \geq 1$ and $f(z)$ be a sense-preserving homeomorphism of $D=\{z|r \leq| z \mid \leq 1\}$ onto $D_{1}=\left\{w\left|r^{1 / K} \leq\right| w \mid \leq 1\right\}$. If

$\bmod A \leq K \bmod f(A) \quad$ for any annulus $A=A\left(r_{1}, r_{2}\right) \subset A$, then $|f(z)|=|z|^{1 / K}$ for any $z \in D$. 
Proof. Let $A^{\prime}=\{z|r<| z \mid<R\}$ and $A^{\prime \prime}=\{z|R<| z \mid<1\}$. Then by Lemma 2,

$$
\begin{aligned}
\frac{1}{2 K \pi} \log \frac{1}{r} & =\frac{1}{K} \bmod D=\frac{1}{K}\left(\bmod A^{\prime}+\bmod A^{\prime \prime}\right) \\
& \leq \bmod f\left(A^{\prime}\right)+\bmod f\left(A^{\prime \prime}\right) \leq \bmod D_{1}=\frac{1}{2 K \pi} \log \frac{1}{r} .
\end{aligned}
$$

Hence

$$
\bmod f\left(A^{\prime}\right)+\bmod f\left(A^{\prime \prime}\right)=\bmod D_{1},
$$

as well as

$$
\bmod f\left(A^{\prime}\right)=\frac{\bmod A^{\prime}}{K} \text { and } \bmod f\left(A^{\prime \prime}\right)=\frac{\bmod A^{\prime \prime}}{K} .
$$

Thus by Lemma $3, f\left(A^{\prime}\right)=\left\{w\left|r^{1 / K}<\right| w \mid<R^{1 / K}\right\}$ and $f\left(A^{\prime \prime}\right)=\{z \mid$ $\left.R^{1 / K}<|z|<1\right\}$. This completes the proof.

In the same way as in the proof of Theorem 2, from Corollary 1, we obtain

Corollary 2. Let $K \geq 1$ and $f(z)$ be a sense-preserving homeomorphism of $D=\{z|r \leq| z \mid \leq 1\}$ onto $D_{1}=\left\{w\left|r^{1 / K} \leq\right| z \mid \leq 1\right\}$. If $\bmod A \leq K \bmod f(A) \quad$ for any annulus $A=A\left(r_{1}, r_{2}\right) \subset D$,

and

$$
\bmod f(A) \leq \frac{1}{K} \bmod A \quad \text { for any } A=A\left(r_{1}, r_{2} ; \theta_{1}, \theta_{2}\right) \subset D,
$$

then $f(z)=\lambda z|z|^{1 / K-1}$ for any $z \in D$, where $\lambda$ is a constant with $|\lambda|=1$.

The following problem was suggested by Chen in [3].

Problem 2. Let $f(z)$ be a sense-preserving homeomorphism of the unit disk $\Delta$ onto itself with $f(0)=0$. If for some $K \geq 1$,

$$
\bmod f(A) \leq K \bmod A \quad \text { for any } A=A\left(r_{1}, r_{2} ; \theta_{1}, \theta_{2}\right) \subset \Delta \text {, }
$$

and

$$
\lim _{z \rightarrow 0} \frac{|f(z)|}{|z|^{K}}=1
$$

is it true that $f(z)=\lambda z|z|^{K-1}$ for any $z \in \Delta$, where $\lambda$ is a constant with $|\lambda|=1$ ?

We cannot give an answer to this problem although we think it is positive. But Theorem 3 settles a weak form of the above problem.

Proof of Theorem 3. Without loss of generality, we assume that $f(1)=1$. Let $A_{1}=A(r, 1 ; 0, \theta)$ and $A_{2}=A(r, 1 ; \theta, 2 \pi)$. Then

$$
\frac{1}{\bmod A_{1}}+\frac{1}{\bmod A_{2}}=\frac{1}{\bmod D_{1}}=\frac{2 \pi}{\log \frac{1}{r}},
$$


and

$$
\frac{1}{\bmod f\left(A_{i}\right)} \geq \frac{1}{K} \frac{1}{\bmod A_{i}}, \quad i=1,2 .
$$

By Lemma 2,

$$
\frac{1}{\bmod f\left(A_{1}\right)}+\frac{1}{\bmod f\left(A_{2}\right)} \leq \frac{1}{\bmod D_{2}}=\frac{1}{K} \frac{2 \pi}{\log \frac{1}{r}} .
$$

Then

$$
\begin{aligned}
\frac{1}{K} \frac{2 \pi}{\log \frac{1}{r}} & =\frac{1}{K}\left(\frac{1}{\bmod A_{1}}+\frac{1}{\bmod A_{2}}\right) \\
& \leq \frac{1}{\bmod f\left(A_{1}\right)}+\frac{1}{\bmod f\left(A_{2}\right)} \leq \frac{1}{K} \frac{2 \pi}{\log \frac{1}{r}}
\end{aligned}
$$

Hence

$$
\frac{1}{\bmod f\left(A_{1}\right)}+\frac{1}{\bmod f\left(A_{2}\right)}=\frac{1}{K} \frac{2 \pi}{\log \frac{1}{r}},
$$

as well as

$$
\bmod f\left(A_{i}\right)=K \bmod A_{i}, \quad i=1,2 .
$$

From Lemma 3 it follows that $f\left(A_{1}\right)=A\left(r^{K}, 1 ; 0, \theta\right)$ and $f\left(A_{2}\right)=$ $A\left(r^{K}, 1 ; \theta, 2 \pi\right)$. This means that $f(z)$ maps the radius $\left\{z=k e^{i \theta} \mid r \leq k \leq 1\right\}$ onto the radius $\left\{w=k^{K} e^{i \theta} \mid r \leq k \leq 1\right\}$ for any $0 \leq \theta \leq 2 \pi$.

Next we prove that $|f(z)|=|z|^{K}$ for every $z \in D_{1}$. Indeed, suppose that there exists $z_{0} \in A$ such that $\left|f\left(z_{0}\right)\right| \neq\left|z_{0}\right|^{K}$. We assume that $\left|f\left(z_{0}\right)\right|<\left|z_{0}\right|^{K}$ (the case where $\left|f\left(z_{0}\right)\right|>\left|z_{0}\right|^{K}$ can be treated similarly). Let $\varepsilon=\left|z_{0}\right|^{K}-$ $\left|f\left(z_{0}\right)\right|>0$ and $z_{0}=r_{0} e^{i \theta_{0}}$. Then by the continuity of $f(z)$, there exists $\delta>0$ such that $|z|^{K}-|f(z)| \geq \varepsilon / 2$ for any $z \in\left\{z=r_{0} e^{i\left(\theta_{0}+\theta\right)} \mid-\delta \leq \theta \leq \delta\right\}$. Let $A_{0}=A\left(\left|z_{0}\right|, 1 ; \theta_{0}-\delta, \theta_{0}+\delta\right)$. By Lemma 1 and the above facts,

$$
\begin{aligned}
\bmod f\left(A_{0}\right) & \geq \bmod A\left(\left|z_{0}\right|^{K}-\varepsilon / 2,1 ; \theta_{0}-\delta, \theta_{0}+\delta\right) \\
& >\bmod A\left(\left|z_{0}\right|^{K}, 1 ; \theta_{0}-\delta, \theta_{0}+\delta\right)=K \bmod A_{0},
\end{aligned}
$$

a contradiction.

Combining the above facts, we obtain the conclusion.

From Theorem 3 we can conjecture that the answer to Problem 2 should be positive.

Another proof of Theorem A. It is obvious that $f(z)$ satisfies the conditions of Theorem 1 , so $|f(z)|=|z|^{1 / K}$. Take $g=f^{-1}: \Delta \rightarrow \Delta$. For any $0<r \leq 1$, let $D_{r}=\{z|r \leq| z \mid \leq 1\}$. Then $g: D_{r} \rightarrow D_{r}$ satisfies the conditions of Theorem 3, so $g(z)=\lambda z|z|^{K-1},|\lambda|=1$. Because $r$ is arbitrarily chosen, we conclude that $f(z)=\lambda z|z|^{1 / K-1}$ on $\Delta$.

Because the inverse of a $K$-quasiconformal mapping is still $K$-quasiconformal, we have: 
COROLlary 3. If $K \geq 1$ and $f(z)$ is a K-quasiconformal mapping of $\Delta$ onto itself which satisfies $f(0)=0$ and

$$
\lim _{z \rightarrow 0} \frac{|f(z)|}{|z|^{K}}=1
$$

then $f(z)=\lambda z|z|^{K-1}$ for any $z \in \Delta$, where $\lambda$ is a constant with $|\lambda|=1$.

It is natural to pose the following problem:

Problem 3. Let $f(z)$ be a sense-preserving homeomorphism of the unit disk $\Delta$ onto itself, with $f(0)=0$. Suppose that for some $K \geq 1$,

$$
\bmod f(A) \leq K \bmod A \quad \text { for any } A=A\left(r_{1}, r_{2} ; \theta_{1}, \theta_{2}\right) \subset \Delta .
$$

Is then $f(z)$ a quasiconformal mapping?

We give a negative answer to this problem by the following example:

ExAmple 3 . Let $f: \Delta \rightarrow \Delta$ be defined by the equality $f(z)=r^{K_{1}(1+t r)} e^{i \theta}$ for $z=r e^{i \theta}$, where $t$ is a positive constant. By direct computation, its Beltrami coefficient is

$$
\mu\left(r e^{i \theta}\right)=\frac{\left(-1+K_{1}\right)+K_{1} \operatorname{tr}(1+\log r)}{\left(1+K_{1}\right)+K_{1} \operatorname{tr}(1+\log r)} e^{2 i \theta} .
$$

If we choose $r$ and $t$ such that $1+\operatorname{tr}(1+\log r)=0$, then $\mid \mu\left(r e^{i \theta} \mid=1\right.$. So $f(z)$ is not quasiconformal.

Next we show that for this example, (5) holds with $K=K_{1}+K_{1} t$. By symmetry and the definition of the modulus of sectors, it is sufficient to show that for any fixed $t>0$, there exists $K>0$ such that for any $0<r_{1}<r_{2} \leq 1$,

$$
\frac{\log \frac{r_{2}^{K_{1}\left(1+t r_{2}\right)}}{r_{1}^{K_{1}\left(1+t r_{1}\right)}}}{\log \frac{r_{2}}{r_{1}}} \leq K .
$$

Let $h(r)=r \log r-\log r$. Then $h^{\prime}(r)=1+\log r-1 / r$ for $0<r \leq 1$. Hence $h^{\prime \prime}(r)=1 / r+1 / r^{2} \geq 0$ for $0<r \leq 1$. Because $h^{\prime}(1)=0, h^{\prime}(r) \leq 0$ for any $0<r \leq 1$. So $h(r)$ is decreasing when $0<r \leq 1$. Thus for $0<r_{1}<r_{2} \leq 1$, $r_{2} \log r_{2}-\log r_{2} \leq r_{1} \log r_{1}-\log r_{1}$. Consequently,

$$
\frac{r_{2} \log r_{2}-r_{1} \log r_{1}}{\log r_{2}-\log r_{1}} \leq 1
$$

Let $g(r)=r \log r+\log r$. Then $g^{\prime}(r)=1+\log r+1 / r$ and $g^{\prime \prime}(r)=$ $1 / r-1 / r^{2} \leq 0$ for $0<r \leq 1$. As $g^{\prime}(1)=2$ we know that $g^{\prime}(r)>0$ for $0<r \leq 1$. So $g(r)$ is increasing for $0<r \leq 1$. Thus for $0<r_{1}<r_{2} \leq 1$, $r_{2} \log r_{2}+\log r_{2} \geq r_{1} \log r_{1}+\log r_{1}$. This implies that

$$
\frac{r_{2} \log r_{2}-r_{1} \log r_{1}}{\log r_{2}-\log r_{1}} \geq-1 \text {. }
$$


Combining the above, we have

$$
K_{1}-K_{1} t \leq \frac{\log \frac{r_{2}^{K_{1}\left(1+t r_{2}\right)}}{r_{1}^{K_{1}\left(1+t r_{1}\right)}}}{\log \frac{r_{2}}{r_{1}}} \leq K_{1}+K_{1} t=K .
$$

So if we take $t>0$, the mapping $f(z)$ satisfies (5) with $K=K_{1}+K_{1} t$, but is not quasiconformal. This gives a negative answer to Problem 3.

Moreover, Example 3 shows that the answer to the following problem is negative.

Problem 4. Let $f(z)$ be a sense-preserving homeomorphism of the unit disk $\Delta$ onto itself, with $f(0)=0$. Suppose that for some $K \geq 1$, $\bmod A \leq K \bmod f(A) \quad$ for any $A=A\left(r_{1}, r_{2} ; \theta_{1}, \theta_{2}\right) \subset \Delta$.

Is $f(z)$ a quasiconformal mapping?

Acknowledgements. The authors deeply appreciate the referee's very careful reading of our paper and many helpful suggestions.

\section{References}

[1] L. V. Ahlfors, Lectures on Quasiconformal Mappings, Van Nostrand, Princeton, NJ, 1966.

[2] —, Conformal Invariants, McGraw-Hill, New York, 1979.

[3] Z. Chen, Geometric characterization for affine mappings and Teichmüller mappings, Studia Math. 157 (2003), 71-82.

[4] F. W. Gehring and J. Välsälä, On the geometric definition for quasiconformal mappings, Comment. Math. Helv. 36 (1962), 19-32.

[5] O. Lehto and K. I. Virtanen, Quasiconformal Mappings in the Plane, Springer, Berlin, 1973.

[6] Z. Li, Quasiconformal Mapping and its Applications in the Theory of Riemann Surfaces, Science Press, Beijing, 1988.

[7] H. Zhu, Z. Zhou and C. He, The characterization of Grötzsch's problem in a domain, J. Fudan Univ. 38 (1999), 205-207.

Shulong Li

School of Biomedical Engineering

Southern Medical University

Guangzhou 510515, P.R. China
Lixin Liu

Department of Mathematics

Sun Yat-sen University

Guangzhou 510275, P.R. China

E-mail: mcsllx@mail.sysu.edu.cn

Received June 21, 2006

Revised version May 15, 2008 